\title{
Monoclonal Antibody hLEU 2A
}

National Cancer Institute

\section{Source}

National Cancer Institute. Monoclonal Antibody hLEU 2A. NCI Thesaurus. Code C29176.

A recombinant monoclonal antibody directed against the human suppressor-cytotoxic $\mathrm{T}$ cell subset and designed to deplete circulating Leu 2A-positive lymphocytes. The antigen LEU 2A, also known as CD8, is present on a subset of natural killer (NK) lymphocytes as well as the on the human suppressor/cytotoxic T-lymphocyte subset. (NCI04) 